\title{
EQUILIBRIUM STATES OF INTERMEDIATE ENTROPIES
}

\section{PENG Sun}

\author{
China Economics and Management Academy \\ Central University of Finance and Economics \\ Beijing 100081, China
}

\begin{abstract}
We explore an approach to the conjecture of Katok on intermediate entropies that based on uniqueness of equilibrium states, provided the entropy function is upper semi-continuous. As an application, we prove Katok's conjecture for Mañé diffeomorphisms.
\end{abstract}

\section{INTRODUCTION}

Let $(X, d)$ be a compact metric space and $f: X \rightarrow X$ be a continuous map. Denote by $\mathcal{M}(f)$ the subspace of all invariant measures for the dynamical system $(X, f)$ and by $\mathcal{M}_{e}(f)$ the subset of all ergodic measures. Denote by $C(X)$ the space of all continuous potentials on $X$, equipped with the $C^{0}$ (supremum) norm $\|\cdot\|$. For $\phi \in C(X)$, denote by $P(\phi)=P(f, \phi)$ the topological pressure of $(X, f, \phi)$ and by $P_{\mu}(\phi)=P_{\mu}(f, \phi):=h_{\mu}(f)+\int \phi d \mu$ the pressure of $\mu \in \mathcal{M}(f)$. We usually omit $f$ when it is clearly fixed. The Variational Principle states that

$$
P(\phi)=\sup \left\{P_{\mu}(\phi): \mu \in \mathcal{M}(f)\right\} .
$$

For convenience, we say that $(X, f)$ is a $U S C$ system if the entropy function $\mu \mapsto$ $h_{\mu}(f)$ is upper semi-continuous, where $h_{\mu}(f)$ denotes the metric entropy of $(X, f)$ with respect to $\mu \in \mathcal{M}(f)$. Denote the set of equilibrium states for $(X, f, \phi)$ by

$$
\mathcal{E}(\phi):=\left\{\mu \in \mathcal{M}(f): P_{\mu}(\phi)=P(\phi)\right\} .
$$

It is well-known that when $(X, f)$ is a USC system, $\mathcal{E}(\phi)$ is nonempty for every $\phi \in C(X)$. Study on equilibrium states has a long history. Existence, uniqueness and properties of equilibrium states are important and popular topics in dynamical systems. For example, see [1, 2, 3, 7, 8, 16].

As indicated in [24, Section 9.4], The topological pressure $P(f, \cdot)$ for the system $(X, f)$, as a functional on continuous potentials, determines all invariant probability measures and their entropies. In this article, we would like to further explore such connections and show its application to the conjecture of Katok on intermediate entropies.

Denote

$$
\mathscr{P}(f, \phi):=\left\{P_{\mu}(f, \phi): \mu \in \mathcal{M}_{e}(f)\right\}
$$

2010 Mathematics Subject Classification. Primary: 37A35, 37D35.

Key words and phrases. equilibrium state, intermediate entropy, pressure, Mañé diffeomorphism, ergodic optimization, thermodynamical formalism. 
and

$$
\mathscr{H}(f):=\mathscr{P}(f, 0)=\left\{h_{\mu}(f): \mu \in \mathcal{M}_{e}(f)\right\} .
$$

We say that $(X, f)$ has the intermediate entropy property if $\mathscr{H}(f) \supset[0, h(f))$, i.e. for every $a \in[0, h(f))$, there is an ergodic measure $\mu \in \mathcal{M}_{e}(f)$ such that $h_{\mu}(f)=a$.

Conjecture (Katok). Let $(X, f)$ be a $C^{2}$ diffeomorphism on a compact Riemannian manifold. Then $(X, f)$ has the intermediate entropy property.

Partial results on Katok's conjecture have been obtained in [10, 17, 18, 23, 19, 15, 6, 12, 13, 21, 20, 22]. They represent two major approaches, which are based on hyperbolic structures and specification-like properties, respectively. What we illustrate here is a new one, which shows that for USC systems, certain results on equilibrium states imply the intermediate entropy property. This approach is inspired by some facts in ergodic optimization.

Denote

$$
\mathscr{U}(f):=\{\phi \in C(X):(X, f, \phi) \text { has a unique equilibrium state }\} .
$$

For $\phi \in C(X)$, denote by $\mathcal{M}_{\max }(f, \phi)$ the set of maximizing measures for $\phi$, i.e. invariant measures $\mu \in \mathcal{M}(f)$ that maximizes $\int \phi d \mu$.

Theorem 1.1. Let $(X, f)$ be a USC system. Suppose that there is $\phi \in C(X)$ such that:

(1) $t \phi \in \mathscr{U}(f)$ for every $t \geq 0$.

(2) $\mathcal{M}_{\max }(f, \phi)=\left\{\mu_{\phi}\right\}$ is a singleton and $h_{\mu_{\phi}}(f)=0$.

Then $(X, f)$ has the intermediate entropy property.

In fact, the ergodic measures of intermediate entropies we obtain in Theorem 1.1 are just the unique equilibrium states for $t \phi$, whose metric entropy varies continuously with $t$. When $\mathcal{M}_{\max }(f, \phi)=\left\{\mu_{\phi}\right\}$ is a singleton, $\mu_{\phi}$ is the unique ground state and the zero temperature limit of these equilibrium states.

We shall prove a more general result than Theorem 1.1. We can relax the uniqueness of equilibrium states and conclude on intermediate pressures. For $\psi \in$ $C(X)$, let $\mathscr{V}(f, \psi)$ be the set of all continuous potentials $\phi$ such that $P_{\mu}(\psi)$ is a constant, denoted by $P^{\psi}(\phi)$, for all $\mu \in \mathscr{E}(\phi)$. That is,

$$
\mathscr{V}(f, \psi):=\left\{\phi \in C(X): P_{\mu}(\psi)=P^{\psi}(\phi) \text { for every } \mu \in \mathcal{E}(\phi)\right\} .
$$

In particular, $\mathscr{V}(f):=\mathscr{V}(f, 0)$ is the set of all potentials whose equilibrium states have equal entropies. By definition, we have $\psi \in \mathscr{V}(f, \psi)$ and $\mathscr{U}(f) \subset \mathscr{V}(f, \psi)$ for every $\psi \in C(X)$.

Theorem 1.2. Let $(X, f)$ be a USC system. Suppose that there are $\psi, \phi \in C(X)$ and $\alpha \in \mathbb{R}$ such that the following holds:

(1) $\psi+t \phi \in \mathscr{V}(f, \psi)$ for every $t \geq 0$.

(2) $P_{\mu}(\psi) \leq \alpha$ for every $\mu \in \mathcal{M}_{\max }(f, \phi)$.

Then $\mathscr{P}(f, \psi) \supset[\alpha, P(\psi)]$.

Theorem 1.1 and 1.2 apply to the Mañé diffeomorphisms considered in [4] and [22]. The following theorem completely verifies Katok's conjecture for such systems, which improves [22, Corollary 1.2]. See Section 4 for details.

Theorem 1.3. Mañé diffeomorphisms have the intermediate entropy property. 


\section{Continuity of Equilibrium States}

Readers are also referred to [24] for definitions and basic properties of entropies and pressures. The following lemma is a simple observation (cf. [16, 6.8]).

Lemma 2.1. For any system $(X, f)$ and any $\phi, \psi \in C(X)$, we have

$$
|P(\phi)-P(\psi)| \leq\|\phi-\psi\| \text { for any } \phi, \psi \in C(X) .
$$

So $P(\cdot)$ is a (Lipschitz) continuous function on $C(X)$.

We try to make our results as more applicable as possible. Proposition 2.2 and 2.3 are slight variations of known results (cf. [11, Theorem 4.2.11] and [9, Theorem 4.1]). In particular, (1) and (6) (hence Proposition 2.2 and Proposition 2.3) hold when the measures are actually the equilibrium states for the corresponding potentials, i.e. $\mu_{n} \in \mathcal{E}\left(\phi_{n}\right)$ or $\mu_{n} \in \mathcal{E}\left(\psi+t_{n} \phi_{n}\right)$ for all $n$.

Proposition 2.2. Let $(X, f)$ be a USC system. Let $\left\{\phi_{n}\right\}_{n=1}^{\infty}$ be a sequence of continuous potentials such that $\left\|\phi_{n}-\phi\right\| \rightarrow 0$. Let $\left\{\mu_{n}\right\}_{n=1}^{\infty}$ be a sequence in $\mathcal{M}(f)$ such that $\mu_{n} \rightarrow \mu$ and

$$
\lim _{n \rightarrow \infty}\left|P_{\mu_{n}}\left(\phi_{n}\right)-P\left(\phi_{n}\right)\right|=0 .
$$

Then $\mu$ is an equilibrium state for $\phi$, i.e. $\mu \in \mathcal{E}(\phi)$.

Proof. As $P(\cdot)$ is continuous, we have $P\left(\phi_{n}\right) \rightarrow P(\phi)$. So (1) is equivalent to the condition

$$
\lim _{n \rightarrow \infty}\left|P_{\mu_{n}}\left(\phi_{n}\right)-P(\phi)\right|=0
$$

We fix a metric $D$ on $\mathcal{M}(f)$ that induces the weak-* topology. For every $\varepsilon>0$, as the entropy map is upper semi-continuous, there is $\eta_{\varepsilon}>0$ such that for every $\nu \in B\left(\mu, \eta_{\varepsilon}\right)$ we have

$$
h_{\nu}(f)<h_{\mu}(f)+\varepsilon \text { and }\left|\int \phi d \nu-\int \phi d \mu\right|<\varepsilon .
$$

By (2), for every $\varepsilon>0$, there is $N$ such that

$$
\left\|\phi_{N}-\phi\right\|<\varepsilon, D\left(\mu_{N}, \mu\right)<\eta_{\varepsilon} \text { and } P_{\mu_{N}}\left(\phi_{N}\right)>P(\phi)-\varepsilon \text {. }
$$

Hence by (3), we have

$$
h_{\mu}(f)>h_{\mu_{N}}(f)-\varepsilon \text { and }\left|\int \phi d \mu_{N}-\int \phi d \mu\right|<\varepsilon .
$$

By (4) and (5), we have

$$
\begin{aligned}
P_{\mu}(\phi) & =h_{\mu}(f)+\int \phi d \mu \\
& >h_{\mu_{N}}(f)-\varepsilon+\int \phi d \mu_{N}-\left|\int \phi d \mu_{N}-\int \phi d \mu\right| \\
& >h_{\mu_{N}}(f)-\varepsilon+\left(\int \phi_{N} d \mu_{N}-\left|\int \phi_{N} d \mu_{N}-\int \phi d \mu_{N}\right|\right)-\varepsilon \\
& >P_{\mu_{N}}\left(\phi_{N}\right)-\left\|\phi_{N}-\phi\right\|-2 \varepsilon \\
& >P(\phi)-4 \varepsilon .
\end{aligned}
$$

This implies that $P_{\mu}(\phi) \geq P(\phi)$ as it holds for all $\varepsilon>0$. Hence $\mu$ is an equilibrium state for $\phi$. 
Proposition 2.3. Let $(X, f)$ be any system and $\psi, \phi \in C(X)$. Let $\left\{t_{n}\right\}_{n=1}^{\infty}$ be a sequence of real numbers such that $t_{n} \rightarrow \infty$. Let $\left\{\mu_{n}\right\}_{n=1}^{\infty}$ be a sequence in $\mathcal{M}(f)$ such that $\mu_{n} \rightarrow \mu$ and

$$
\lim _{n \rightarrow \infty} \frac{1}{t_{n}}\left|P_{\mu_{n}}\left(\psi+t_{n} \phi\right)-P\left(\psi+t_{n} \phi\right)\right|=0 .
$$

Then $\mu$ is a maximizing measure for $\phi$, i.e. $\mu \in \mathcal{M}_{\max }(\phi)$.

Proof. For every $\varepsilon>0$, there is $\eta_{\varepsilon}>0$ such that for every $\nu \in B\left(\mu, \eta_{\varepsilon}\right)$ we have

$$
\left|\int \psi d \nu-\int \psi d \mu\right|<\varepsilon \text { and }\left|\int \phi d \nu-\int \phi d \mu\right|<\varepsilon
$$

As $t_{n} \rightarrow \infty, \mu_{n} \rightarrow \mu$, by (6), for every $\varepsilon>0$, there is $N$ such that for all $n>N$ we have

$$
t_{n} \varepsilon>h(f), D\left(\mu_{n}, \mu\right)<\eta_{\varepsilon} \text { and } P\left(\psi+t_{n} \phi\right)-P_{\mu_{n}}\left(\psi+t_{n} \phi\right)<t_{n} \varepsilon
$$

By (7) and (8), for all $n>N$, we have

$$
\begin{aligned}
\left|P_{\mu}(\psi)-P_{\mu_{n}}(\psi)\right| & \leq\left|h_{\mu}(f)-h_{\mu_{n}}(f)\right|+\left|\int \psi d \mu-\int \psi d \mu_{n}\right| \\
& <h(f)+\varepsilon<\left(t_{n}+1\right) \varepsilon
\end{aligned}
$$

and hence

$$
\begin{aligned}
& P\left(\psi+t_{n} \phi\right)-P_{\mu}\left(\psi+t_{n} \phi\right) \\
\leq & P\left(\psi+t_{n} \phi\right)-P_{\mu_{n}}\left(\psi+t_{n} \phi\right)+\left|P_{\mu}\left(\psi+t_{n} \phi\right)-P_{\mu_{n}}\left(\psi+t_{n} \phi\right)\right| \\
< & \left|P_{\mu}(\psi)-P_{\mu_{n}}(\psi)\right|+t_{n}\left|\int \phi d \mu-\int \phi d \mu_{n}\right|+t_{n} \varepsilon \\
< & \left(3 t_{n}+1\right) \varepsilon .
\end{aligned}
$$

By (9) and (10), for every $\nu \in \mathcal{M}(f)$ and all $n>N$, we have

$$
\begin{aligned}
& t_{n}\left(\int \phi d \mu-\int \phi d \nu\right) \\
= & \left(P_{\mu}\left(\psi+t_{n} \phi\right)-P_{\mu}(\psi)\right)-\left(P_{\nu}\left(\psi+t_{n} \phi\right)-P_{\nu}(\psi)\right) \\
> & \left(P\left(\psi+t_{n} \phi\right)-P_{\nu}\left(\psi+t_{n} \phi\right)\right)-\left(P\left(\psi+t_{n} \phi\right)-P_{\mu}\left(\psi+t_{n} \phi\right)\right) \\
& -\left|P_{\mu}(\psi)-P_{\nu}(\psi)\right| \\
> & -\left(4 t_{n}+1\right) \varepsilon .
\end{aligned}
$$

As $t_{n} \rightarrow \infty$, (11) implies that

$$
\int \phi d \mu \geq \int \phi d \nu-4 \varepsilon \text { for every } \nu \in \mathcal{M}(f) .
$$

This implies that $\int \phi d \mu \geq \int \phi d \nu$ as (12) holds for all $\varepsilon>0$. Hence $\mu$ is a maximizing measure for $\phi$.

\section{Intermediate Pressures}

Proposition 3.1. Let $(X, f)$ be a USC system and $\psi \in C(X)$. Then the function $\phi \mapsto P^{\psi}(\phi)$ is continuous on $\mathscr{V}(f, \psi)$. 
Proof. Let $\left\{\phi_{n}\right\}_{n=1}^{\infty}$ be a sequence in $\mathscr{V}(f, \psi)$ such that $\phi_{n} \rightarrow \tilde{\phi} \in \mathscr{V}(f, \psi)$. For each $n$, take any $\mu_{n} \in \mathscr{E}\left(\phi_{n}\right)$. Let $\mu$ be the limit of a convergent subsequence $\left\{\mu_{n_{k}}\right\}_{k=1}^{\infty}$. As $\phi_{n_{k}} \rightarrow \tilde{\phi}$, by Proposition 2.2, we have $\mu \in \mathscr{E}(\tilde{\phi})$.

By Lemma 2.1, we have

$$
\begin{aligned}
& \lim _{k \rightarrow \infty}\left|P^{\psi}\left(\phi_{n_{k}}\right)-P^{\psi}(\tilde{\phi})\right| \\
= & \lim _{k \rightarrow \infty}\left|P_{\mu_{n_{k}}}(\psi)-P_{\mu}(\psi)\right| \\
\leq & \lim _{k \rightarrow \infty}\left(\left|P_{\mu_{n_{k}}}\left(\phi_{n_{k}}\right)-P_{\mu}(\tilde{\phi})\right|+\left|\int\left(\phi_{n_{k}}-\psi\right) d_{\mu_{n_{k}}}-\int(\tilde{\phi}-\psi) d_{\mu}\right|\right) \\
\leq & \lim _{k \rightarrow \infty}\left|P\left(\phi_{n_{k}}\right)-P(\tilde{\phi})\right|+\lim _{k \rightarrow \infty}\left|\int\left(\phi_{n_{k}}-\tilde{\phi}\right) d_{\mu_{n_{k}}}\right| \\
& +\lim _{k \rightarrow \infty}\left|\int(\tilde{\phi}-\psi) d_{\mu_{n_{k}}}-\int(\tilde{\phi}-\psi) d_{\mu}\right| \\
\leq & \lim _{k \rightarrow \infty}\left\|\phi_{n_{k}}-\tilde{\phi}\right\|+\lim _{k \rightarrow \infty}\left\|\phi_{n_{k}}-\tilde{\phi}\right\|+0 \\
= & 0 .
\end{aligned}
$$

This implies that $P^{\psi}(\cdot)$ is a continuous function on $\mathscr{V}(f, \psi)$.

Corollary 3.2. Let $(X, f)$ be a USC system. Denote by $\mu_{\phi}$ the unique equilibrium state for each $\phi \in \mathscr{U}(f)$. Then the map $\phi \mapsto h_{\mu_{\phi}}(f)$ is continuous on $\mathscr{U}(f)$.

Proof. Note that $\mathscr{U}(f) \in \mathscr{V}(f)$ and $P^{0}(\phi)=P_{\mu_{\phi}}(0)=h_{\mu_{\phi}}(f)$ for every $\phi \in \mathscr{U}(f)$. Apply Proposition 3.1 for $\psi=0$.

Proposition 3.3. Let $(X, f)$ be a USC system and $\psi \in C(X)$. Suppose that there is a continuous path $\Phi:[0, T] \rightarrow \mathscr{V}(f, \psi)$ and $P_{\psi}(\Phi(0))<P_{\psi}(\Phi(T))$. Then

$$
\mathscr{P}(f, \psi) \supset\left[P^{\psi}(\Phi(0)), P^{\psi}(\Phi(T))\right]
$$

Proof. By Proposition 3.1, $P^{\psi}(\cdot)$ is continuous on $\mathscr{V}(f, \psi)$. Hence $P^{\psi} \circ \Phi$ is continuous. Then (13) follows from the Intermediate Value Theorem.

Analogous to Corollary 3.2, we have:

Corollary 3.4. Let $(X, f)$ be a USC system. Suppose that there is a continuous path $\Phi:[0, T] \rightarrow \mathscr{U}(f)$, i.e. $\Phi(t)$ has a unique equilibrium state $\mu_{t}$ for each $t \in[0, T]$. Assume that $h_{\mu_{0}}(f) \leq h_{\mu_{T}}(f)$. Then we have

$$
\mathscr{H}(f) \supset\left[h_{\mu_{0}}(f), h_{\mu_{T}}(f)\right]
$$

Proof of Theorem 1.2. Take $\mu_{n} \in \mathcal{E}(\psi+t \phi)$ for each $n \in \mathbb{N}$. As $\mathcal{M}(f)$ is compact, there is a subsequence $\left\{t_{k}\right\}_{k=1}^{\infty}$ such that $t_{k} \rightarrow \infty$ and $\left\{\mu_{t_{k}}\right\}_{k=1}^{\infty}$ converges to $\mu \in$ $\mathcal{M}(f)$. By Proposition 2.3, $\mu$ is a maximizing measure for $\phi$ and hence $P_{\mu}(\psi) \leq a$. For any $b \in(a, P(\psi))$, as $\mu_{t_{k}} \rightarrow \mu$ and the entropy map is upper semi-continuous, there is $N$ such that $P_{\mu_{t_{N}}}(\psi)<b$. Note that $\Phi(t):=\psi+t \phi$ is a continuous path from $\left[0, t_{N}\right]$ to $\mathscr{V}(f, \psi)$. By Proposition 3.3, we have

$$
\mathscr{P}(\psi) \supset(b, P(\psi)] .
$$

Then $\mathscr{P}(\psi) \supset[a, P(\psi)]$ because $P_{\mu}(\psi) \leq a$ and (14) holds for any $b \in(a, P(\psi))$.

Corollary 3.5. Let $(X, f)$ be a USC system with positive topological entropy $h(f)>$ 0 . Suppose that there is a continuous potential $\phi$ such that the following holds: 
(1) $(X, f, t \phi)$ has a unique equilibrium state for every $t \geq 0$.

(2) $(X, f, \phi)$ has a unique maximizing measure $\mu$ with $h_{\mu}(f)=0$.

Then $(X, f)$ has the intermediate entropy property.

Proof. Note that for $t=0, \mu_{0}$ is just the measure of maximal entropy. Hence $h_{\mu_{0}}(f)=h(f)$. Apply Theorem 1.2 for $\psi=0$.

It is well known that every Axiom A system has unique equilibrium states for Hölder potentials [1]. One can just pick a fixed point $p$ and take $\phi(x):=-d(x, p)$ for every $x \in X$, which is a Hölder function with the unique maximizing measure supported on $p$. In this case Corollary 3.5 provides another proof that every Axiom A system has the intermediate entropy property.

\section{Application to Mañé Diffeomorphisms}

Following [4], we consider the Mañé family $\mathfrak{M}_{\rho, r}$, which is a class of DA (derived from Anosov) maps first introduced by Mañé [14]. They are $C^{0}$ perturbations of a hyperbolic toral automorphism $f_{A}: \mathbb{T}^{d} \rightarrow \mathbb{T}^{d}$, which are partially hyperbolic with 1-dimensional centers. Let $q$ be a fixed point of $f_{A}$. As described in [4], for each $g \in \mathfrak{M}_{\rho, r}$ we assume that:

(1) $\rho>0$ such that the neighborhood $B(q, \rho)$ is the support of the perturbation, i.e. $g=f$ on $\mathbb{T}^{3} \backslash B(q, \rho)$.

(2) $r \in[0,1]$ such that if an orbit of $g$ spends a proportion at least $r$ of its time outside $B(q, \rho)$, then it contracts the vectors in the central direction.

(3) The $C^{0}$ distance between $g$ and $f_{A}$ is sufficiently small, i.e. there is a constant $\eta=\eta\left(f_{A}\right)$ depending only on $f_{A}$ such that $d_{C^{0}}\left(g, f_{A}\right)<\eta$. In particular, this holds when $\rho$ is sufficiently small.

For $\phi \in C\left(\mathbb{T}^{d}\right), \rho, L>0$ and $r \in(0,1)$, denote

$$
\Xi(\rho, r, \phi, L):=(1-r) \sup _{B(q, \rho)} \phi+r\left(\sup _{\mathbb{T}^{d}} \phi+h\left(f_{A}\right)+L\right)+H(2 r),
$$

where

$$
H(r):=-r \ln r-(1-r) \ln (1-r) .
$$

Denote

$$
\mathscr{C}_{\rho, r, g, L}:=\left\{\phi \in C\left(\mathbb{T}^{d}\right): P(g, \phi)>\Xi(\rho, r, \phi, L)\right\}
$$

Let $\phi$ be an $\alpha$-Hölder potential on $\mathbb{T}^{d}$ and

$$
|\phi|_{\alpha}:=\sup \left\{\frac{|\phi(x)-\phi(y)|}{d(x, y)^{\alpha}}: x, y \in X, x \neq y\right\}
$$

be its Hölder semi-norm. Denote

$$
\mathscr{C}_{M}^{\alpha}:=\left\{\phi \in C\left(\mathbb{T}^{d}\right):|\phi|_{\alpha}<M\right\} .
$$

Theorem 4.1 ([4, Theorem A and B]). Let $g \in \mathfrak{M}_{\rho, r}$ and $\phi$ be an $\alpha$-Hölder continuous function on $\mathbb{T}^{3}$.

(1) There is a constant $L=L\left(f_{A}\right)$ depending only on $f_{A}$ such that $\left(\mathbb{T}^{d}, g, \phi\right)$ has a unique equilibrium state as long as $\phi \in \mathscr{C}_{\rho, r, g, L}$.

(2) There is a function $M(\rho, r)$ such that $M(\rho, r) \rightarrow \infty$ as $\rho, r \rightarrow 0$ and $\left(\mathbb{T}^{d}, g, \phi\right)$ has a unique equilibrium sate as long as $\phi \in \mathscr{C}_{M(\rho, r)}^{\alpha}$.

Let $p$ be another fixed point of $f_{A}$ such that $p \notin \overline{B(q, \rho)}$. We can fix $\Psi \in C\left(\mathbb{T}^{d}\right)$ such that 
(1) $\Psi(p)=0$.

(2) $\Psi(x)=-1$ for every $x \in B(q, \rho)$.

(3) $\Psi(x)<0$ for every $x \in \mathbb{T}^{d} \backslash\{p\}$.

(4) $\Psi$ is $\alpha$-Hölder.

Denote by $\mu_{p}$ the Dirac measure on $p$. Then $\mu_{p}$ is the unique maximizing measure for $\Psi$ and $h_{\mu_{p}}(g)=0$.

Lemma 4.2. There are $\rho^{*}>0$ and $r^{*} \in(0,1)$ such that for every $g \in \mathfrak{M}_{\rho^{*}, r^{*}}$, we have $t \Psi \in \mathscr{U}(g)$ for all $t \geq 0$.

Proof. Denote

$$
\beta_{r}:=\frac{r\left(h\left(f_{A}\right)+L\right)+H(2 r)}{1-r} .
$$

Note that $\beta_{r} \rightarrow 0$ as $r \rightarrow 0$. By Theorem 4.1(2), there are $\rho^{*}>0$ and $r^{*} \in(0,1)$ such that

$$
\beta_{r^{*}}|\Psi|_{\alpha}<M\left(\rho^{*}, r^{*}\right)
$$

This implies that for every $g \in \mathfrak{M}_{\rho^{*}, r^{*}}$, we have

$$
t \Psi \in \mathscr{C}_{M\left(\rho^{*}, r^{*}\right)}^{\alpha} \subset \mathscr{U}(g) \text { for every } t \in\left[0, \beta_{r^{*}}\right] .
$$

For $t>\beta_{r^{*}}$, as $h_{\mu_{p}}(g)=0$, by the Variational Principle, we have

$$
\begin{aligned}
\Xi\left(\rho^{*}, r^{*}, t \Psi, L\right) & =(1-r)(-t)+r\left(h\left(f_{A}\right)+L\right)+H(2 r) \\
& <0 \\
& =h_{\mu_{p}}(g)+\int(t \Psi) d \mu_{p} \\
& \leq P(g, t \Psi) .
\end{aligned}
$$

By Theorem 4.1(1), for every $g \in \mathfrak{M}_{\rho^{*}, r^{*}}$, we have

$$
t \Psi \in \mathscr{C}_{\rho^{*}, r^{*}, g, L} \subset \mathscr{U}(g) \text { for every } t>\beta_{r^{*}} .
$$

Remark. We may choose $\Psi$ such that $|\Psi|_{\alpha}$ is as small as possible to achieve larger values of $\rho^{*}$ and $r^{*}$ as in Lemma 4.2.

Theorem 4.3. For every $g \in \mathfrak{M}_{\rho^{*}, r^{*}}$, the system $\left(\mathbb{T}^{d}, g\right)$ has the intermediate entropy property.

Proof. By [5, Proposition 6], every Mañé diffeomorphism is entropy expansive. So $\left(\mathbb{T}^{d}, g\right)$ is a USC system. As $\mu_{p}$ is the unique maximizing measure for $\Psi$ and $h_{\mu_{p}}(g)=0$, the conclusion follows from Lemma 4.2 and Corollary 3.5.

\section{ACKNOWLEDGMENTS}

The author is supported by National Natural Science Foundation of China (No. 11571387) and CUFE Young Elite Teacher Project (No. QYP1902). The author learned about ergodic optimization from Yiwei Zhang and Yun Yang, and would like to thank them for fruitful discussions. 


\section{REFERENCES}

[1] R. Bowen, Some systems with unique equilibrium states, Math. Systems Theory 8 (1974/75), no. 3, 193-202.

[2] B. Bowen Equilibrium states and the ergodic theory of Anosov diffeomorphisms, revised ed., Lecture Notes in Mathematics, vol. 470, Springer-Verlag, Berlin, 2008, With a preface by David Ruelle, Edited by Jean-René Chazottes.

[3] V. Climenhaga and D. J. Thompson, Unique equilibrium states for flows and homeomorphisms with non-uniform structure. Adv. Math. 303 (2016), 744-799.

[4] V. Climenhaga, T. Fisher and D. J. Thompson, Equilibrium states for Mañé diffeomorphisms. Ergodic Theory and Dynamical Systems, 2018, 1-23. doi:10.1017/etds.2017.125

[5] W. Cowieson and L.-S. Young, SRB measures as zero-noise limits. Ergod. Th. \& Dynam. Sys. 25(4) (2005), 1115-1138.

[6] L. Guan, P. Sun and W. Wu, Measures of Intermediate Entropies and Homogeneous Dynamics, Nonlinearity, 30 (2017), 3349-3361.

[7] M. Denker, G. Keller, M. Urbański, On the uniqueness of equilibrium states for piecewise monotone mappings, Studia Math. 1990, 97(1), 27-36.

[8] G. Iommi, M. Todd, Natural equilibrium states for multimodal maps, Comm. Math. Phys. 2010, 300(1), 65-94.

[9] O. Jenkinson, Ergodic optimization in dynamical systems, Ergod. Th. \& Dynam. Sys. 2019, 39, 2593-2618.

[10] A. Katok and L. Mendoza, Dynamical systems with nonuniformly hyperbolic behavior, supplement to Introduction to the Modern Theory of Dynamical Systems, by A. Katok and B. Hasselblatt, Cambridge University Press, 1995.

[11] G. Keller, Equilibrium States in Ergodic Theory, Cambridge University Press, 1998.

[12] J. Konieczny, M. Kupsa, D. Kwietniak, Arcwise connectedness of the set of ergodic measures of hereditary shifts. Proceedings of the American Mathematical Society, 2018, 146(8), 34253438.

[13] J. Li and P. Oprocha, Properties of invariant measures in dynamical systems with the shadowing property. Ergod. Th. \& Dynam. Sys. 2018, 38, 2257-2294.

[14] R. Mañé, Contributions to the stability conjecture, Topology, 17 (1978), 383-396.

[15] A. Quas, and T. Soo, Ergodic universality of some topological dynamical systems, Transactions of the American Mathematical Society, 2016, 368(6), 4137-4170.

[16] D. Ruelle, Thermodynamic formalism. Encyclopedia of Mathematics and its Applications, 5. Addison-Wesley Publishing Co., Reading, Mass., 1978.

[17] P. Sun, Zero-entropy invariant measures for skew product diffeomorphisms, Ergodic Theory and Dynamical Systems, 30 (2010), 923-930.

[18] P. Sun, Measures of intermediate entropies for skew product diffeomorphisms, Discrete Contin. Dyn. Syst - A, 2010, 27(3), 1219-1231.

[19] P. Sun, Density of metric entropies for linear toral automorphisms, Dynamical Systems, 2012, 27(2), 197-204.

[20] P. Sun, Zero-entropy dynamical systems with gluing orbit property. preprint, 2019, arXiv:1810.08980.

[21] P. Sun, Ergodic measures of intermediate entropies for dynamical systems with approximate product property. preprint, 2019, arXiv:1906.09862.

[22] P. Sun, Denseness of intermediate pressures for systems with the ClimenhagaThompson structures. Journal of Mathematical Analysis and Applications, 487(2), 2020, doi:10.1016/j.jmaa.2020.124027.

[23] R. Ures, Intrinsic ergodicity of partially hyperbolic diffeomorphisms with a hyperbolic linear part. Proceedings of the American Mathematical Society, 2012, 140(6), 1973-1985.

[24] P. Walters, An Introduction to Ergodic Theory, Springer-Verlag, 1982.

E-mail address: sunpeng@cufe.edu.cn 